\title{
CHONDROGENICALLY DIFFERENTIATED MESENCHYMAL STROMAL CELL PELLETS STIMULATE ENDOCHONDRAL BONE REGENERATION IN CRITICAL-SIZED BONE DEFECTS
}

\author{
J. van der Stok ${ }^{1, *}$, M.K.E. Koolen ${ }^{2}$, H. Jahr ${ }^{3}$, N. Kops ${ }^{1}$, J.H. Waarsing ${ }^{1}$, H. Weinans ${ }^{2,4,5}$ and O.P. van der Jagt ${ }^{1}$
}

${ }^{1}$ Orthopaedic Research Laboratory, Department of Orthopaedics, Erasmus MC, University Medical Centre Rotterdam, Rotterdam, the Netherlands

${ }^{2}$ Department of Orthopaedics, Utrecht University Medical Centre, Utrecht, the Netherlands

${ }^{3}$ Department of Orthopaedic Surgery, University Hospital RWTH Aachen, Aachen, Germany

${ }^{4}$ Department of Biomechanical Engineering, Delft University of Technology, Delft, the Netherlands

${ }^{5}$ Department of Rheumatology, Utrecht University Medical Centre, Utrecht, the Netherlands

\begin{abstract}
Grafting bone defects or atrophic non-unions with mesenchymal stromal cells (MSCs)-based grafts is not yet successful. MSC-based grafts typically use undifferentiated or osteogenically differentiated MSCs and regenerate bone through intramembranous ossification. Endochondral ossification might be more potent but requires chondrogenic differentiation of MSCs. Here, we determined if chondrogenically differentiated MSC (ch-MSC) pellets could induce bone regeneration in an orthotopic environment through endochondral ossification. Undifferentiated MSC pellets (ud-MSC) and ch-MSC pellets were generated from MSCs of human donors cultured on chondrogenic medium for respectively 3 (ud-MSC) and 21 (ch-MSC) days. A $6 \mathrm{~mm}$ femoral bone defect was made and stabilised with an internal plate in 27 athymic rats. Defects were left empty for 6 weeks to develop an atrophic non-union before they were grafted with ch-MSC pellets or ud-MSC pellets. Micro-CT scans made 4 and 8 weeks after grafting showed that ch-MSC pellets resulted in significantly more bone than ud-MSC pellets. This regenerated bone could completely bridge the defect, but the amount of bone regeneration was donordependent. Histology after 7 and 14 days showed slowly mineralising pellets containing hypertrophic chondrocytes, as well as TRAP-positive and CD34-positive cells around the ch-MSC pellets, indicating osteoclastic resorption and vascularisation typical for endochondral ossification. In conclusion, grafting critical femoral bone defects with chondrogenically differentiated MSC pellets led to rapid and pronounced bone regeneration through endochondral ossification and may therefore be a more successful MSCbased graft to repair large bone defects or atrophic nonunions. But, since bone regeneration was donor-depend, the generation of potent chondrogenically differentiated MSC pellets for each single donor needs to be established first.
\end{abstract}

Keywords: Bone defect; bone regeneration; critical size defect; endochondral ossification; fracture healing; mesenchymal stromal cells; non-union.

*Address for correspondence:

Johan van der Stok

Orthopaedic Research Laboratory

Department of Orthopaedics, Erasmus MC

Room Ee1614, PO Box 2040

3000CA Rotterdam, The Netherlands
Telephone Number: +31-10-7043384

FAX Number: +31-10-7044690

Email: j.vanderstok@erasmusmc.nl

\section{Introduction}

Bone development and fracture repair occur through intramembranous or endochondral ossification (Shapiro, 2008). In intramembranous ossification, mesenchymal stromal cells (MSCs) differentiate directly into osteoblasts that form bone. In endochondral ossification, MSCs differentiate into chondrocytes, which form a cartilage template (Kronenberg, 2003). Chondrocytes within this template become hypertrophic and start to secrete cytokines such as vascular endothelial growth factor (VEGF), and enzymes like alkaline phosphatase (ALP) and proteases (e.g., matrix metalloproteinases (MMPs)). Together, these factors stimulate vascularisation and mineralisation of the cartilage template, which is subsequently resorbed by attracted osteoclasts and replaced by bone. Which type of ossification predominantly occurs in fracture repair is highly dependent on biomechanical conditions. Absence of motion results in intramembranous ossification, although this is only capable of bridging small gaps (200$500 \mu \mathrm{m}$ ), whereas micromotions stimulate endochondral ossification, through which larger fracture gaps are regenerated (Carter et al., 1998).

Despite modern treatment, fracture repair remains insufficient or impaired in $10 \%$ of all fractures resulting in non-unions or persistent bone defects (Einhorn, 1995). Bone defects and atrophic non-unions can be treated with bone grafts, for which the current gold standard material is autologous bone (Cypher and Grossman, 1996). However, harvesting autologous bone results in a high morbidity rate (10-40 \%) (Banwart et al., 1995; Dimitriou et al., 2011), and the amount that can be harvested is limited. These disadvantages fuelled the development of bone graft substitutes. Ideally a bone graft substitute should consist of a biodegradable material that offers direct mechanical support and functions as an osteoconductive scaffold that facilitates bone regeneration. Also the material should provide osteogenic and angiogenic stimuli to enhance bone regeneration and vascularisation (Giannoudis et al., 2007). Bone graft substitutes currently available for clinical use mainly rely on osteoconductive properties (Van der Stok et al., 2011). Only in a small case-series, an osteoconductive bone substitute has been combined 
with MSCs to graft bone defects (Quarto et al., 2001). But so far the clinical success of MSC-based grafts remains disappointing (Steinert et al., 2012). This may be because those MSC-based grafts contained undifferentiated or osteogenically differentiated MSCs and thus stimulated intramembranous ossification. Differentiation of MSCs towards chondrocytes, and thereby aiming to stimulate endochondral ossification, may be a more successful strategy (Gawlitta et al., 2010).

Chondrogenically differentiated embryonic stem cells (Jukes et al., 2008), embryonic chondrocytes (Oliveira et al., 2009a) or MSCs (Farrell et al., 2009) can undergo hypertrophy and secrete factors similar to those normally secreted by hypertrophic chondrocytes during endochondral ossification (e.g., VEGF, MMP-13, and ALP). In vivo, chondrogenically differentiated MSCs can form bone through endochondral ossification after ectopic implantation in mice (Jukes et al., 2008; Oliveira et al., 2009b; Scotti et al., 2010; Farrell et al., 2011). But ectopic implantation does not fully reflect the complex environment of a bone defect or an atrophic non-union. In an orthotopic environment, bone regeneration starts with a low-grade inflammatory phase (Claes et al., 2012), under low-oxygen tensions (Brighton and Krebs, 1972), and is continuously exposed to biomechanical stimuli. All these factors are known to affect MSCs differentiation and survival (Lacroix and Prendergast, 2002). In this study, we therefore made critical femoral bone defects in rats, and left these defects untreated for 6 weeks to establish an atrophic non-union. Subsequently the defects were grafted with chondrogenically differentiated MSC pellets to determine whether chondrogenically differentiated MSC pellets remain capable of endochondral ossification in this orthotopic environment. We hypothesised that grafting with chondrogenically differentiated MSC pellets enhances bone regeneration and used grafting with undifferentiated MSC pellets as a control. Bone regeneration was evaluated with the use of micro-CT scanning, histology and immunohistochemistry.

\section{Materials and Methods}

Isolation and expansion of human bone marrow cells Human bone marrow derived MSCs of three donors (donor 1: female, 75 years, donor 2: female, 77 years, donor 3: female, 55 years) were obtained during total hip arthroplasty after informed consent and approval of the local medical ethical committee (METC 2004142). Bone marrow aspirates were taken from the great trochanter. Heparinised aspirates were seeded at a density of $30-100 \times 10^{6}$ nucleated cells per T175 flask. After $24 \mathrm{~h}$, non-adherent cells and cell debris were washed out. MSCs were further expanded in low-glucose Dulbecco's modified Eagle medium (DMEM) with $10 \%$ foetal calf serum (Lonza, Basel, Switzerland) supplemented with $50 \mu \mathrm{g} / \mathrm{mL}$ of gentamicin, $1.5 \mu \mathrm{g} / \mathrm{mL}$ of Fungizone (all Invitrogen/Life Technologies, Carlsbad, CA, USA), $0.1 \mathrm{mM}$ of L-ascorbic acid 2-phosphate and $1 \mathrm{ng} / \mathrm{mL}$ of fibroblast growth factor-2 (Instruchemie, Delfzijl, The Netherlands). Cells were cultured at $37{ }^{\circ} \mathrm{C}$ under humidified conditions and
$5 \%$ carbon dioxide $\left(\mathrm{CO}_{2}\right)$. Medium was changed twice a week. When cultured cells neared confluence, they were trypsinised using $0.05 \%$ trypsin and replated at a density of 2,000 cells $/ \mathrm{cm}^{2}$. Cells from the third passage were used for pellet cultures.

\section{Culturing of chondrogenically differentiated MSC pellets and undifferentiated MSC pellets}

After detachment of cells with $0.05 \%$ trypsin, $0.5 \mathrm{~mL}$ of medium, containing 200,000 cells, was put in a polypropylene tube. Tubes were centrifuged for $8 \mathrm{~min}$ at $120 \mathrm{~g}$. Undifferentiated MSC (ud-MSC) pellets and chondrogenically differentiated MSC (ch-MSC) pellets were maintained for respectively 3 and 21 days on high-glucose DMEM, supplemented with $50 \mu \mathrm{g} / \mathrm{mL}$ of gentamicin, $1.5 \mu \mathrm{g} / \mathrm{mL}$ of Fungizone (Invitrogen), $0.1 \mathrm{mM}$ L-ascorbic acid 2-phosphate, $40 \mu \mathrm{g} / \mathrm{mL}$ L-proline, $1 \mathrm{mM}$ of sodium pyruvate (Sigma-Aldrich, St. Louis, MO, USA), 1:100 insulin-transferrin-selenium (ITS) (BD Biosciences, East Rutherford, NJ, USA), $10 \mathrm{ng} / \mathrm{mL}$ of transforming growth factor (TGF)-1 (R\&D Systems, Minneapolis, MN, USA), and $100 \mathrm{nM}$ of dexamethasone (Sigma-Aldrich). After maintaining ud-MSC pellets and ch-MSC pellets for respectively 3 and 21 days, they were directly used for implantation.

\section{Animal experiment}

Twenty-seven skeletally mature male RNU rats (Charles River, Wilmington, MA, USA) were used as experimental animals ( $361 \pm 36 \mathrm{~g}$ body weight). When the animals were 16 weeks old a critical femoral bone defect was made by removal of $6 \mathrm{~mm}$ cortical bone, while stabilised with an internal fixation plate. Six weeks later, in a second surgical procedure, bone defects were grafted with ch-MSC pellets $(n=15)$ or ud-MSC pellets $(n=12)$. The Animal Ethics Committee of the Erasmus University approved the study (ECM 1493) and Dutch guidelines for the care and use of laboratory animals were applied.

\section{Surgical procedures}

Prior to surgery, prophylactic antibiotics (enrofloxacin, $5 \mathrm{mg} / \mathrm{kg}$ body weight) and pain medication (buprenorphine, $0.05 \mathrm{mg} / \mathrm{kg}$ body weight) were administered. Surgical procedures were performed aseptically under general anaesthesia (1-3.5\% isoflurane).

During the first surgical procedure, a critical femoral bone defect was made as follows: the right femur was exposed through a lateral skin incision and division of underlying fascia. A $23 \mathrm{~mm}$ long polyetheretherketone (PEEK) plate was fixed to the anterolateral plane of the femur using three proximal and three distal screws (RatFix, RISystem, Davos-Platz, Switzerland). Periosteum was removed over approximately $8 \mathrm{~mm}$ of the mid-diaphyseal region before removal of $6 \mathrm{~mm}$ cortical bone. Bone was removed with a tailor-made saw guide and a wire. Fascia and skin were sutured in layers using Vicryl 5-0. Antibiotic medication was continued for 2 days post-surgery, and pain medication was continued for 3 days.

During the second surgical procedure, 6 weeks later, critical bone defects were grafted with ch-MSC or udMSC pellets as follows: the right femur was again exposed 


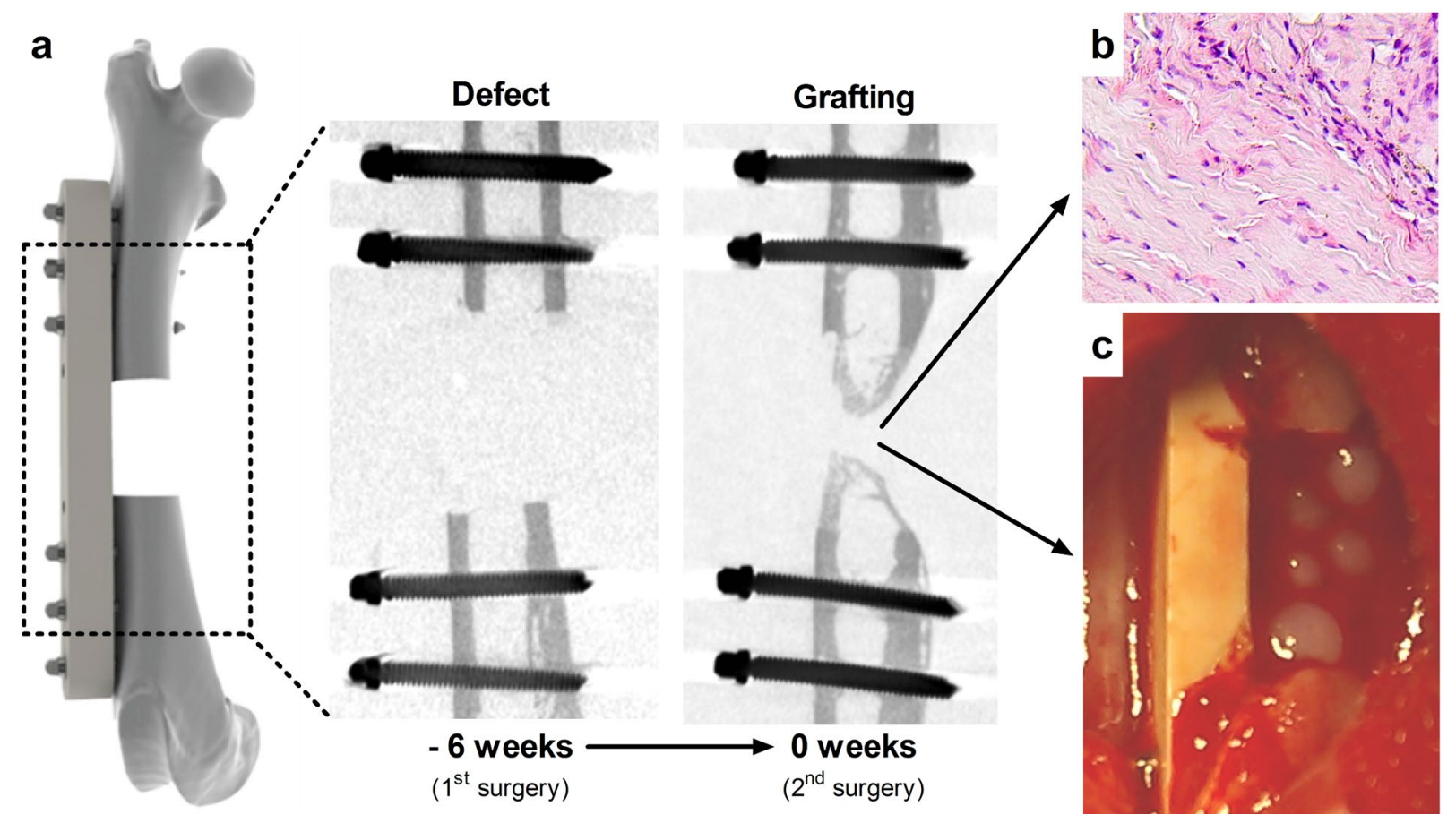

Fig. 1. Critical femur defect with PEEK plate fixation (a) and transversal micro-CT images directly after removal of $6 \mathrm{~mm}$ cortical bone and after 6 weeks. Fibrous tissue that was removed from the defect (b) before 6 ch-MSC pellets were used to graft the defect (c).

through a lateral skin incision and division of underlying fascia. After full exposure of the femur, none of defects had bridged and the defect was filled with fibrous tissue (Fig. 1 $a$ and $b$ ). After debridement and trimming of bone edges at proximal and distal side, defects were grafted with six chMSC or ud-MSC pellets (Fig. 1c). Pellets were contained inside the defect by closure through intramuscular sutures (Vycril 5-0). Subsequently fascia and skin were sutured. Antibiotic medication was continued for two days postsurgery, pain medication was continued for three days. Rats were killed by an overdose pentobarbital.

\section{Micro-CT evaluation}

Bone regeneration was followed longitudinally by in vivo micro-CT scans, acquired under general anaesthesia (1$3.5 \%$ isoflurane). A timeline was acquired by scanning one rat of ch-MSC-group and one rat of ud-MSC-group every week, while the remaining rats ( $n=8$ per group) only underwent scans at 4 and 8 weeks to quantify bone regeneration. Micro-CT scans were made with a SkyScan 1176 scanner (Bruker micro-CT; www.bruker.com) using a $35 \mu \mathrm{m}$-resolution protocol $(65 \mathrm{kV}, 385 \mu \mathrm{A}$ current, $1 \mathrm{~mm} \mathrm{Al}$ filter, and $0.5^{\circ}$ rotation step, resulting in a $7 \mathrm{~min}$ scan). CT images were reconstructed using volumetric reconstruction software NRecon version 1.5 (Bruker micro-CT). Bone volume (BV) was quantified within the $6 \mathrm{~mm}$ defect after segmentation of calcified tissue from non-calcified tissue using a global threshold. This global threshold was determined based on visual inspection and was kept constant for all scans.

\section{Histological and immunohistochemical evaluation}

Histology and immunohistochemistry was done on chMSC and ud-MSC pellets cultured in vitro $(n=3$ per donor) and on grafted femoral bone defects after 3, 7 and 14 days ( $n=2$ ch-MSC-group, $n=1$ ud-MSC-group at each time-point) and after 8 weeks ( $n=8$ per group). Pellets and grafted femora were fixed in $10 \%$ neutral buffered formalin solution for 2 days. Grafted femora were decalcified in $10 \%$ EDTA in phosphate buffered saline (PBS) solution ( $\mathrm{pH}$ 7.4) for 6 weeks. Finally, all specimens were dehydrated in graded ethanol solutions (70-100 \%) and xylene before embedding in paraffin. $6 \mu \mathrm{m}$ paraffin sections were cut with a microtome (Leica, Solms, Germany; RM2135). Pellets were stained with haematoxylin-eosin (H\&E) and thionin, and immunostained for collagen II, collagen $\mathrm{X}$ and vascular endothelial growth factor (VEGF). Grafted femurs were stained with H\&E, thionin, and tartrate-resistant acid phosphatase (TRAP), and immunostained for collagen II and CD34.

Collagen II is a marker for chondrogenic differentiation and collagen $\mathrm{X}$ is a marker for hypertrophy. Antigen retrieval for collagen II was performed through $30 \mathrm{~min}$ incubation of sections with $0.1 \%$ pronase in PBS, while antigen retrieval for collagen $\mathrm{X}$ required $0.1 \%$ pepsin in $0.5 \mathrm{M}$ acetic acid ( $\mathrm{pH} 2.0$ ) for $2 \mathrm{~h}$. Both collagen II and collagen $\mathrm{X}$ stainings continued by incubation with $1 \%$ hyaluronidase in PBS for $30 \mathrm{~min}$. Non-specific binding sites were blocked with $10 \%$ goat serum in PBS and sections were stained overnight with primary antibodies against collagen II (II/II6B3 antibody, 1:100, Developmental 
Studies Hybridoma Bank, Iowa City, IA, USA under contract N01-HD-6-2915 from the National Institute of Child Health and Human Development) or collagen X (1:30, X53, Quartett, Berlin, Germany). To allow the use of monoclonal mouse antibodies on human constructs grafted in a nude rat, we used a method described by Hierck et al. (Hierck et al., 1994). An alkaline-phosphatase-conjugated secondary antibody was used followed by incubation with Neu Fuchsin substrate (Chroma, Rockingham, VT, USA) to demonstrate alkaline-phosphatase activity with a red staining. The positive control for collagen II was bovine cartilage, and for collagen $\mathrm{X}$ we used known hypertrophic pellets. An isotype control was taken along as negative control for both stainings.

VEGF is an angiogenic growth factor, and after microwave treatment in $10 \mathrm{mM}$ citric acid buffer $\mathrm{pH}$ 6.0, sections were blocked with $5 \%$ bovine serum albumin (BSA) in PBS for 10 min and incubated overnight at $4{ }^{\circ} \mathrm{C}$ with VEGF antibody (sc-152, Santa Cruz Biotechnology, Santa Cruz, CA, USA) diluted in $5 \%$ bovine serum albumin (BSA) in PBS. Secondary antibodies against human IgG were conjugated with peroxidase (Dako, Glostrup, Denmark). CD34 is an endothelial cell marker indicating vessel-structures, and antigen retrieval for CD34 was performed through $30 \mathrm{~min}$ incubation with $0.1 \%$ trypsin (Sigma-Aldrich). Non-specific binding sites were blocked with $10 \%$ NGS and sections were incubated with primary antibody against rat CD34 (R\&D Systems). A biotinylated rabbit antibody followed by alkaline phosphatase conjugated streptavidin and incubation with Neu Fuchsin substrate (Chroma) was used to visualise positive staining. Slides were counterstained with haematoxylin. The positive control for VEGF was human skin and an isotype control was taken along as negative control.

\section{Release of angiogenic cytokines}

Secretion of angiogenic cytokines was measured in culture medium taken of the ch-MSC and ud-MSC pellets at the day of implantation using a human cytokine angiogenic multiplex chemiluminescent ELISA (Cat. No. 150251HU, Quansys Biosciences, Logan, UT, USA). This angiogenic multiplex measures angiotensin II (Ang-2), fibroblast growth factor-2 (FGF-2), hepatocyte growth factor (HGF), interleukin-8 (IL-8), platelet derived growth factor-BB (PDGF-BB), tissue inhibitor of metalloproteinase 1 and 2 (TIMP-1 and TIMP-2), tumour necrosis factor- (TNF), and VEGF. Multiplex chemiluminescent ELISA kits were performed according to the manufacturer's instructions and the plate was imaged using Kodak Digital Science ${ }^{\mathrm{TM}}$ Image Station 440CF system (NEN Life Science Products, Boston, MA, USA). Angiogenic cytokine concentrations were calculated using Q-view software ${ }^{\circledR}$ (Quansys Biosciences).

\section{Statistics}

The design of this study was aimed to find at least a duplication in the BV formed within the $6 \mathrm{~mm}$ defect. The standard deviation of BV formed was estimated to be $\sim 50 \%$ based on previous work (Chatterjea et al., 2013; Van der Stok et al., 2013a; Van der Stok et al., 2013b). A simple power calculation using a Students' $t$-test with a $\beta$-value of 0.8 indicates that both groups should consist of a minimum of 5 rats. The final decision to use 8 rats per group accounted for loss of animals (e.g., failure of fixation material to stabilise the defect) or loss of donors (e.g., insufficient of unsuccessful chondrogenic differentiation of MSCs). Statistical analyses were performed using SPSS Statistics 20.0 (SPSS Inc, Chicago, IL, USA). The data are presented as means with standard deviation (SD). Mixed models analysis was used to test for statistical differences between ch-MSC-group and ud-MSC-group, while correcting for donor effect using MSC donor as random factor. A $p$-value less than 0.05 was considered a statistically significant difference.

\section{Results}

\section{Culturing and characterisation of ch-MSC or ud- MSC pellets}

Ch-MSC pellets were 2-3 $\mathrm{mm}$ in diameter and contained hypertrophic chondrocytes surrounded by an extensive extracellular matrix (Fig. 2, a1). This extracellular matrix contained high levels of GAGs, collagen II and collagen $\mathrm{X}$, indicated by thionin staining and collagen II and $\mathrm{X}$ immunostaining (Fig. 2, b1-d1 respectively). VEGF was mainly positive in and around the cells, but not in the extracellular matrix (Fig. 2, e1). Ud-MSC pellets were about $1 \mathrm{~mm}$ in diameter and contained densely packed cells without substantial extracellular matrix (Fig. 2, a2). Cells had not undergone chondrogenic differentiation as no GAGs, nor collagen II or collagen X were observed on thionin staining and collagen II or X immunostaining (Fig. 2 , b2-d2 respectively). VEGF was also produced by udMSC pellets and was mainly found in and around the cells (Fig. 2, e2). Per donor, approximately 180-200 pellets were cultured from passage three human derived MSCs, which can theoretically result in $0.8-2.7 \mathrm{~cm}^{3}$ of ch-MSC pellets.

\section{Micro-CT evaluation}

Two weeks after grafting the defects, ch-MSC pellets started to mineralise whereas no mineralisation of ud-MSC pellets was observed (Fig. 3). Mineralisation of ch-MSC pellets started around the pellets and progressed towards the centre. Mineralised pellets also integrated with bone formed at the cortical bone edges. In one defect grafted with ch-MSC pellets of donor 1, bone formation was capable of completely bridging this defect within 4 weeks (Fig. 4). Once this defect was bridged, the original bone architecture was restored through bone remodelling. In other defects grafted with ch-MSC pellets of donor 1 or donor 3, a minimal gap $(<0.5 \mathrm{~mm})$ remained visible after 8 weeks (Fig. 5a). Defects grafted with ch-MSC pellets of donor 2 or with ud-MSC pellets showed little bone formation and defects were not bridged.

Bone quantification showed that grafting bone defects with ch-MSC pellets led to significantly more bone regeneration than grafting with ud-MSC pellets $(p=0.041)$ (Fig. 5b). After 4 and 8 weeks, average BV of all defects grafted with ch-MSC pellets was almost twice as much as defects grafted with ud-MSC pellets (4 weeks 33.1 


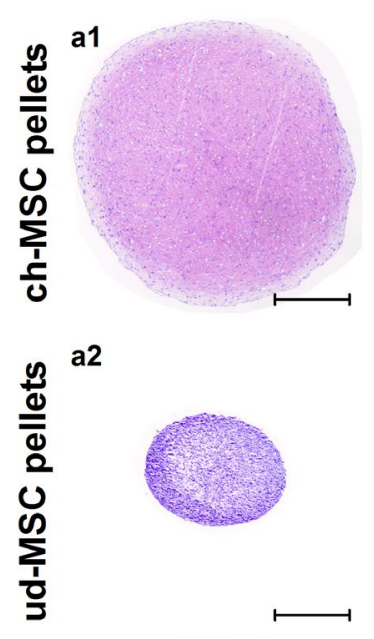

Thionin

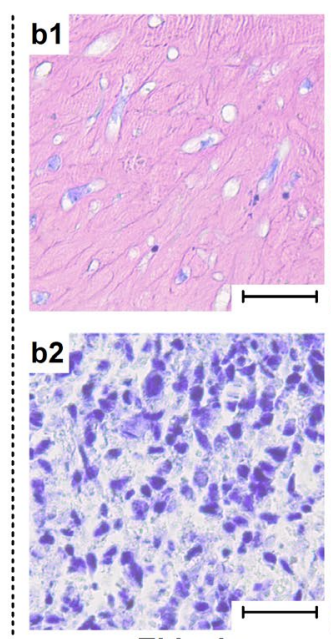

Thionin
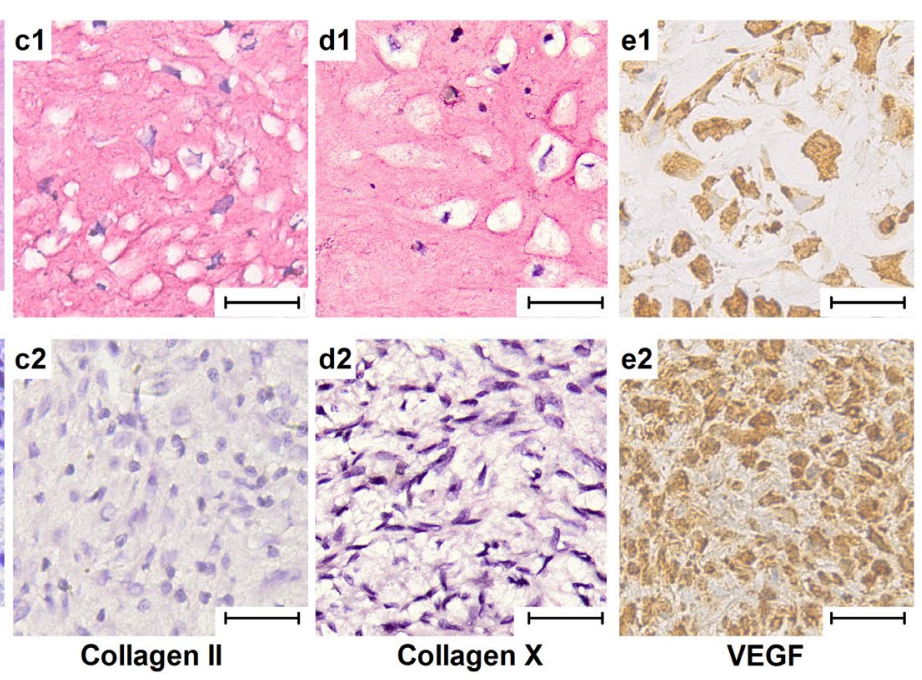

Collagen X

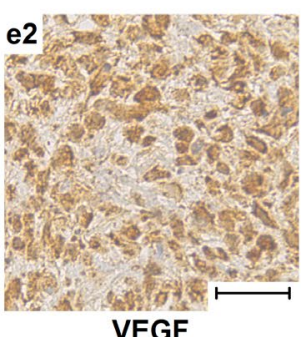

Fig. 2. Histology of ch-MSC pellets (a1) and ud-MSC pellets (a2): ch-MSC pellets were bigger and generated more extracellular matrix than ud-MSC pellets, bar indicates $500 \mu \mathrm{m}$. Detailed views showed that the extracellular matrix of ch-MSC pellets is rich of GAGs (b1), collagen II (c1) and collagen X (d1), indicating that ch-MSC pellets had undergone hypertrophic chondrogenic differentiation. VEGF was predominantly found in and around the cells (e1). Ud-MSC pellets generated little to none extracellular matrix and had not undergone chondrogenic differentiation (b2, c2, and d2). VEGF was found throughout the pellets (e2). Bar indicates $50 \mu \mathrm{m}$.
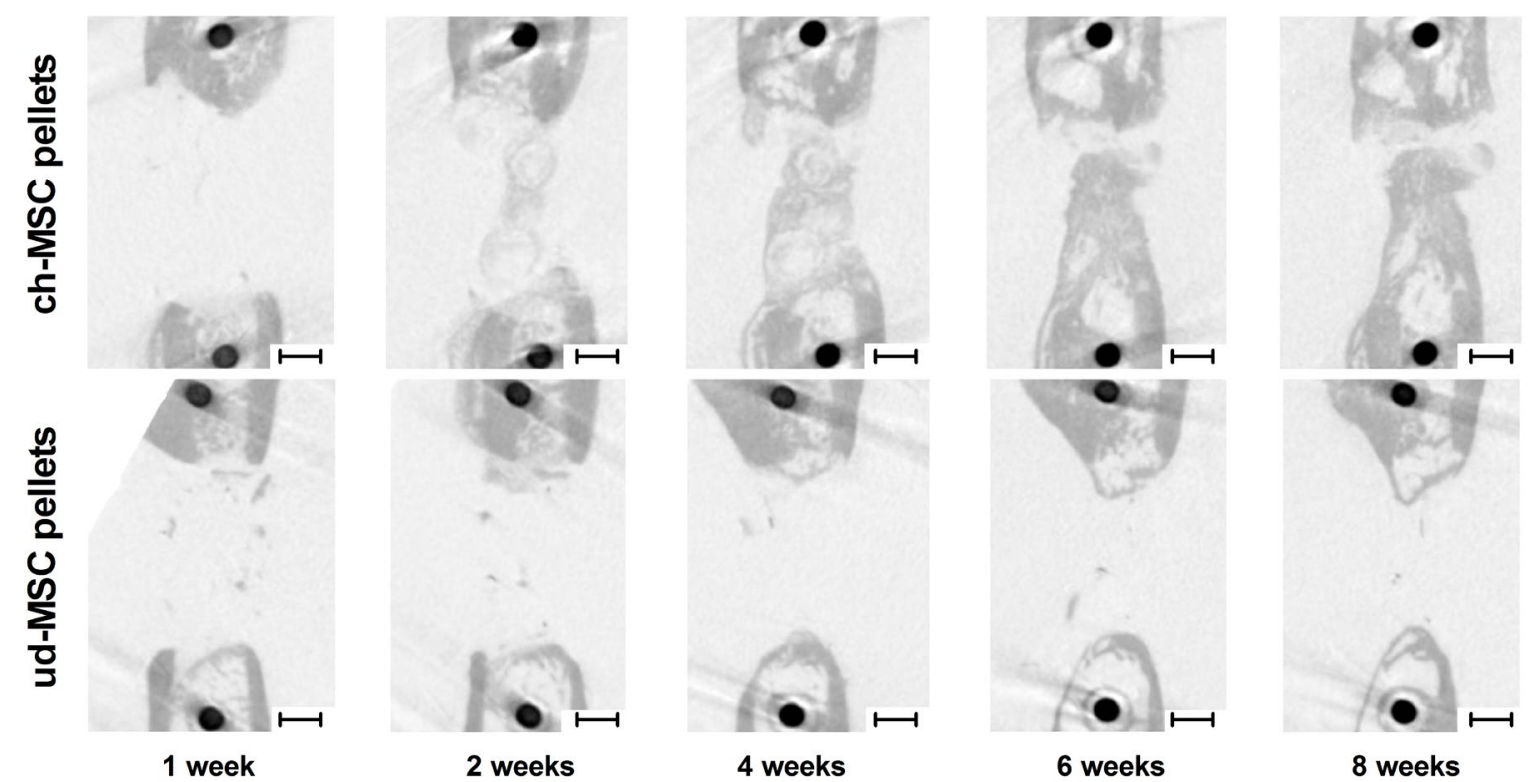

Fig. 3. Repeated micro-CT scans of defects grafted with ch-MSC pellets or ud-MSC pellets of donor 1. Ch-MSC pellets mineralise after 2 weeks, and integrate with adjacent cortical host bone at 4 weeks. Ud-MSC pellets did not show mineralisation throughout the 8 week follow-up. Bar indicates $1 \mathrm{~mm}$.

$\pm 18.8 \mathrm{~mm}^{3}$ versus $17.8 \pm 8.6 \mathrm{~mm}^{3}, 8$ weeks $41.8 \pm 24.7 \mathrm{~mm}^{3}$ versus $24.2 \pm 12.2 \mathrm{~mm}^{3}$ ). This effect was donor dependent, ch-MSC pellets of donor 1 and 3 resulted in BVs of 39.6 $\pm 9.7 \mathrm{~mm}^{3}$ (donor 1 ) and $53.8 \pm 10.7 \mathrm{~mm}^{3}$ (donor 3 ) after 4 weeks and $53.8 \pm 19.5 \mathrm{~mm}^{3}$ (donor 1) and $63.2 \pm 0.8 \mathrm{~mm}^{3}$ (donor 3 ) after 8 weeks. But grafting defects with ch-MSC pellets of donor 2 only resulted in $13.3 \pm 12.4 \mathrm{~mm}^{3} \mathrm{BV}$ after 8 weeks, which was not more than grafting with ud-MSC pellets $\left(25.1 \pm 6.6 \mathrm{~mm}^{3}\right)$. Compared to donor 2, ud-MSC pellets of donor 1 and 3 resulted in similar BVs and were $36.7 \pm 5.2 \mathrm{~mm}^{3}$ and $13.8 \pm 0.1 \mathrm{~mm}^{3}$ respectively.

\section{Histological evaluation}

Grafted ch-MSC pellets were clearly visible in the defect after 7 and 14 days (Fig. 6, a1-2). They formed a cartilage template that bridged the defect. After 7 days, ch-MSC pellets were surrounded by a number of cell layers (Fig. 6, b1, c1, and d1). At this time, some vessel-forming activity was detected around the ch-MSC pellets (Fig. 6, f1: arrows), without substantial osteoclast activity (Fig. 6, e1). After 14 days, the cartilage template was reduced in size (Fig. 6, b1, c1, and d1) and more osteoclast activity was observed along the edges of the remaining ch-MSC pellets (Fig. 6, e2: arrows). Areas of osteoclast activity were 
Fig. 4. Remodelling of mineralised pellets (4 weeks) restoring the original cortex and medullary canal (8 weeks) of the defect grafted with ch-MSC pellets of donor 1 that showed complete bridging. Black arrow heads depict border of ch-MSC pellets, 'c' indicates the newly formed cortex and ' $\mathrm{m}$ ' indicates the ongoing restoration of the medullary canal. Bar indicates $1 \mathrm{~mm}$.

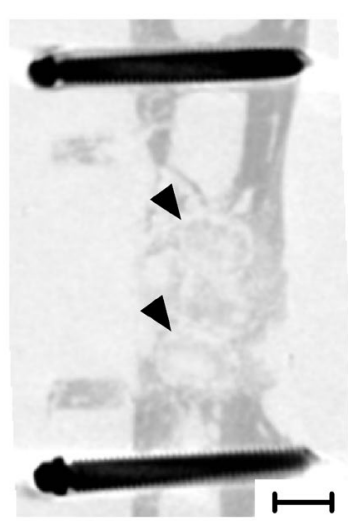

4 weeks

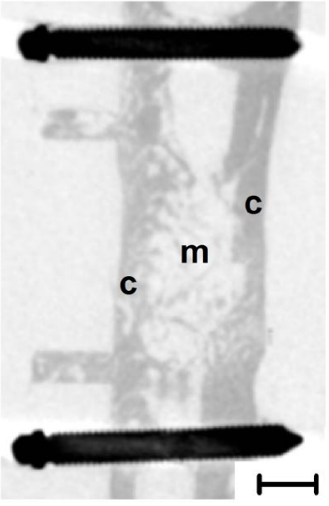

8 weeks a

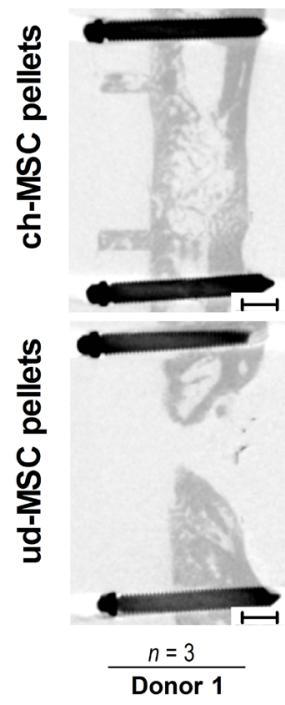

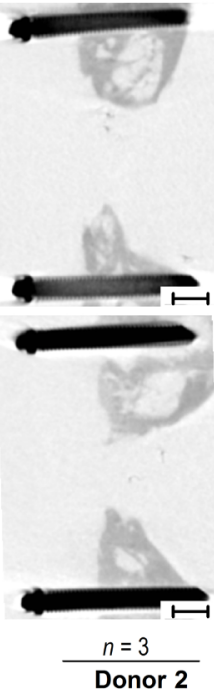
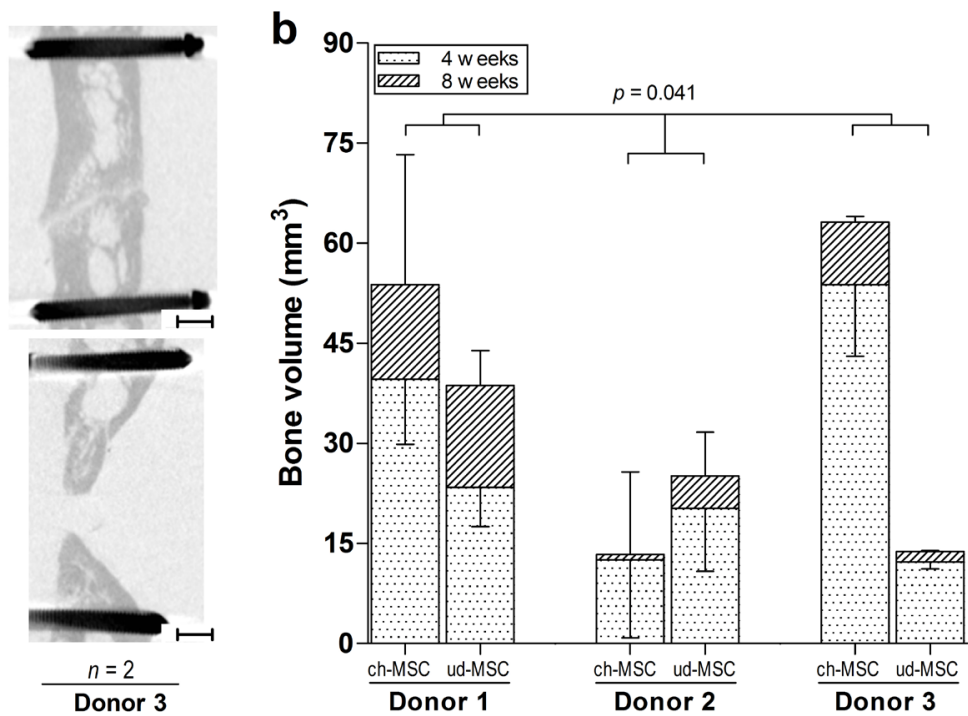

Fig. 5. Micro-CT images of defects grafted with ch-MSC or ud-MSC pellets of all three donors after 8 weeks (a). Defects grafted with ch-MSC pellets of donor 1 and 3 show more bone formation than grafting with ud-MCS pellets, but defects grafted with ch-MSC pellets of donor 2 showed did not show more bone formation than defects grafted ud-MSC pellets of donor 2. Bar indicates $1 \mathrm{~mm}$. Bone volumes measured in $6 \mathrm{~mm}$ bone defect after 4 weeks and 8 weeks (b).
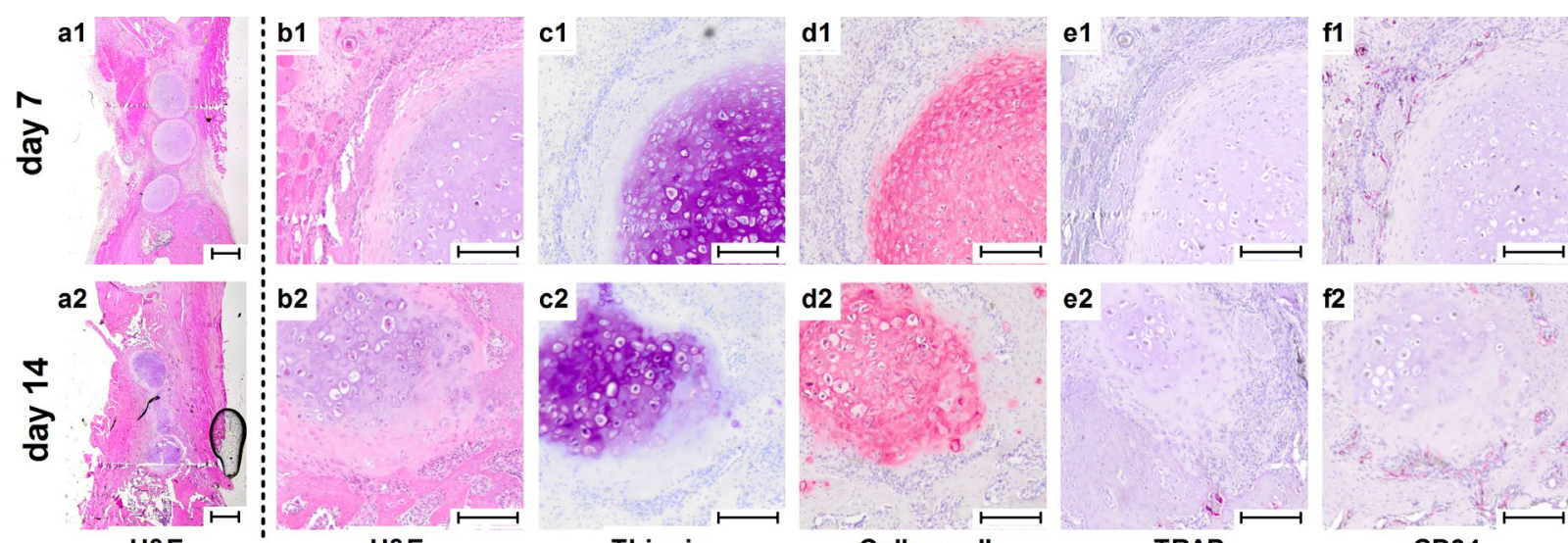

H\&E

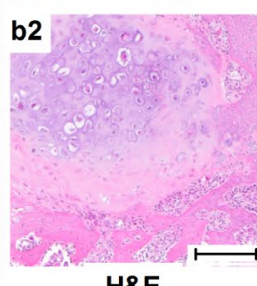

H\&E

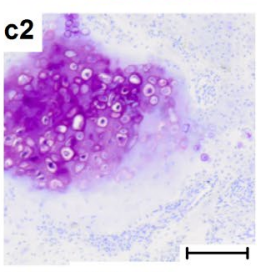

Thionin
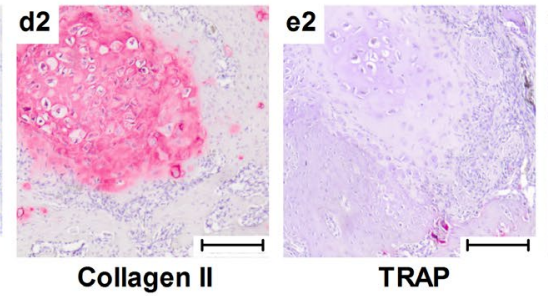

Fig. 6. Histology of the $6 \mathrm{~mm}$ bone defect grafted with ch-MSC pellets after 7 and 14 days (a1-2), bar indicates $500 \mu \mathrm{m}$. Detailed views show that ch-MSC pellets were surrounded by a number of cell layers (b1-2) and that the extracellular matrix remained rich of GAGs (c1-2) and collagen II (d1-2). Osteoclast activity was observed after 14 days (e1-2: arrows) and some vessel-forming activity was found in the surrounding host tissue (f1-2: arrows), bar indicates $250 \mu \mathrm{m}$. 

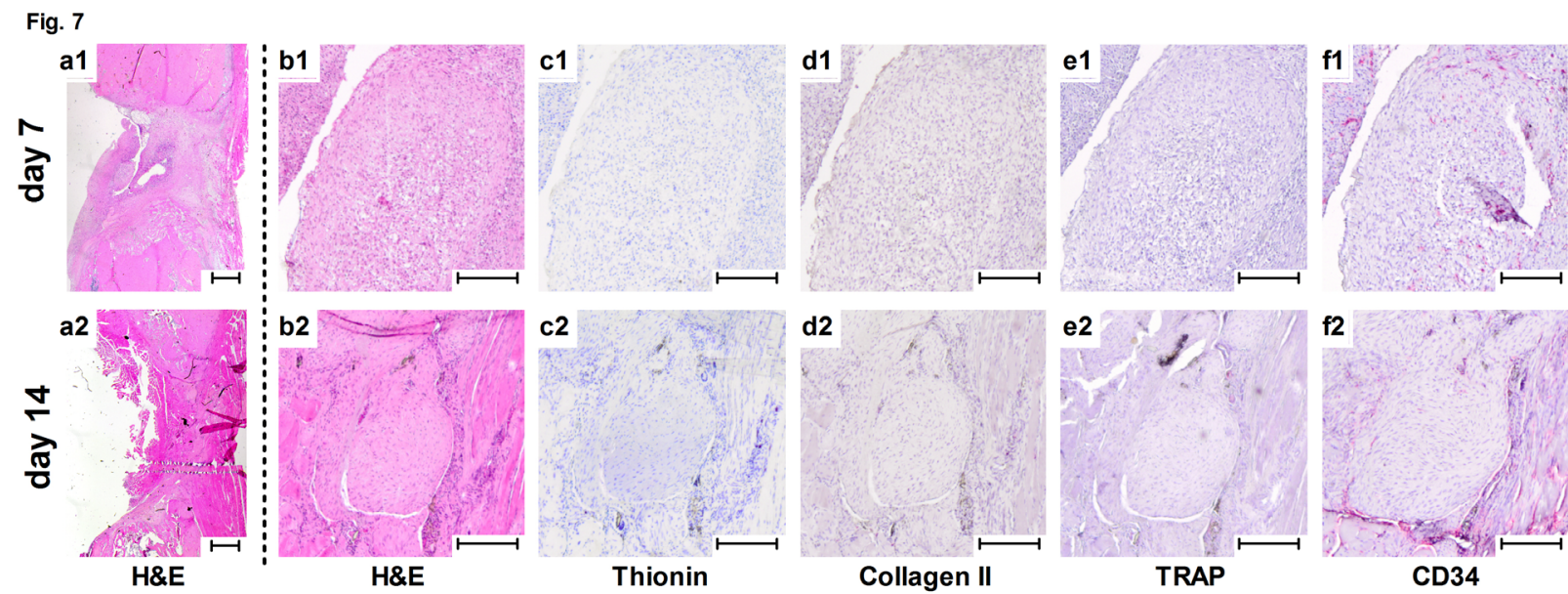

Fig. 7. Histology of the $6 \mathrm{~mm}$ bone defect grafted with ud-MSC pellets after 7 and 14 days (a1-2), bar indicates $500 \mu \mathrm{m}$. Detailed views show that ud-MSC pellets were surrounded by small round cells (b1-2). Ud-MSC pellets did not form an extracellular matrix containing GAGs (c1-2) or collagen II (d1-2), indicating that ud-MSC pellets had not undergone chondrogenic differentiation. No osteoclast activity was observed (e1-2), but widespread vesselforming activity was found in the surrounding host tissue (f1-2: arrows), bar indicates $250 \mu \mathrm{m}$.

Fig. 8. Histology of the $6 \mathrm{~mm}$ bone defect grafted with ch-MSC pellets or ud-MSC pellets after 8 weeks stained with respectively $\mathrm{H} \& \mathrm{E}$ (a1-2) or thionin (b1-2) and corresponding micro-CT images (c1-2). Bone tissue is indicated with ' $b$ ', cartilage tissue is indicated with 'c' and fibrous tissue is indicated with 'f'. Asterisk marks area showed in more detail in Fig. 9. Bar indicates $1 \mathrm{~mm}$.
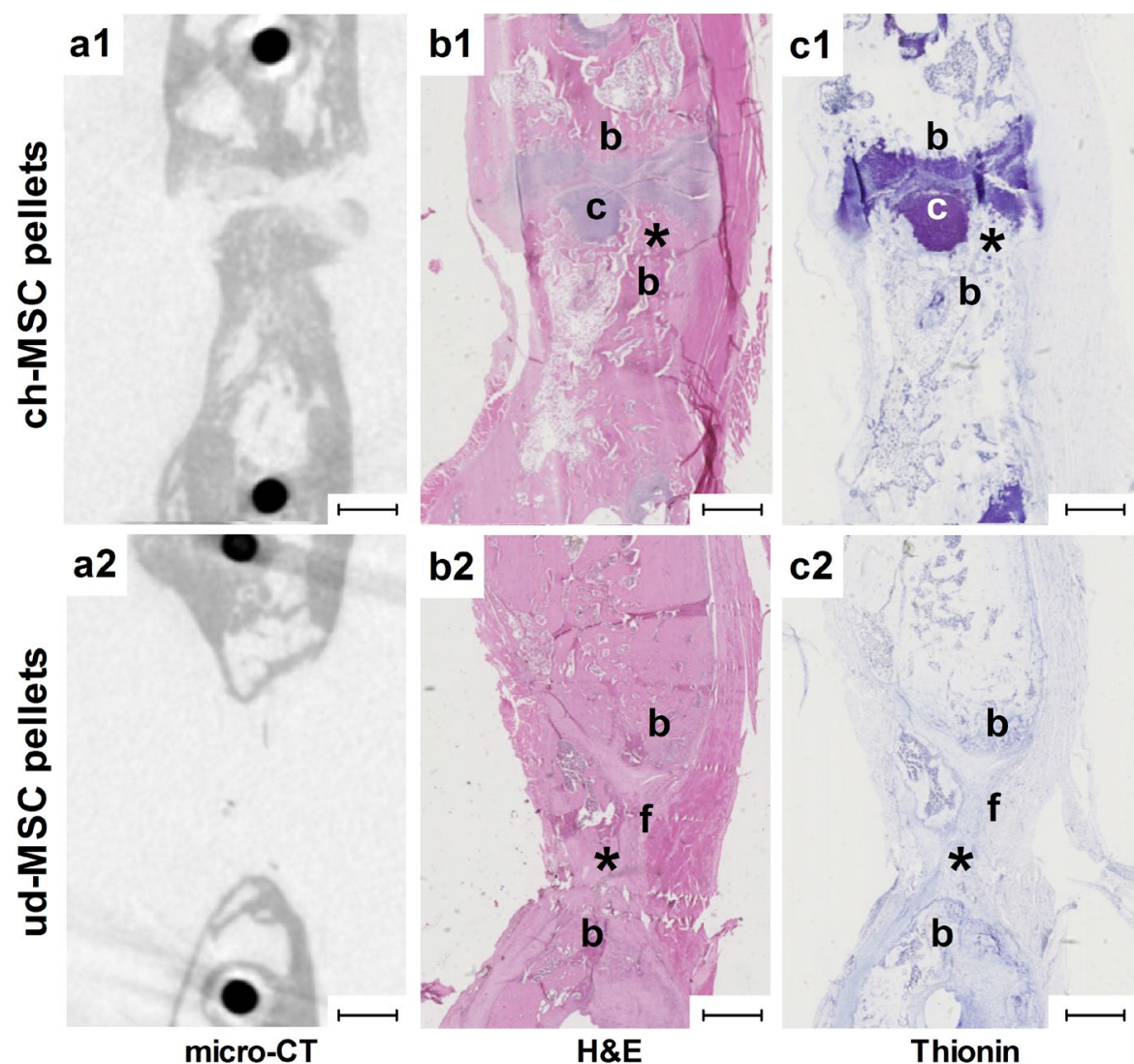

accompanied by vessel-forming activity and vessel-like structures (Fig. 6, f2: arrows). Grafted ud-MSC pellets also remained visible up to 14 days, but did not form a cartilage template inside the defect (Fig. 7, a1-2). After 7 and 14 days, ud-MSC pellets had not undergone chondrogenic differentiation (Fig. 7, b1-2, c1-2 and d1-2) and were surrounded by a high number of small round cells. After 7 and 14 days, there was no osteoclast activity (Fig. 7, e1-2) but in the surrounding tissue substantial vessel-forming activity was observed (Fig. 7, f1-2: arrows).
After 8 weeks, grafted ch-MSC pellets were almost completely resorbed and replaced by bone (Fig. 8, a1 and b1). Bone close to the remaining gap consisted of woven bone and some small areas of vessel-forming activity were found in the proximity of the fracture gap (Fig. 9, a1 and c1: arrows), while the remaining gap consisted of cartilage tissue (Fig 9, b1). In defects grafted with ud-MSC pellets, no cartilage tissue nor remnants of ud-MSC pellets were observed (Fig. 8, a 2 and b2), and the remaining gap was filled with fibrous tissue (Fig. 9, a2 and b2). Within this 

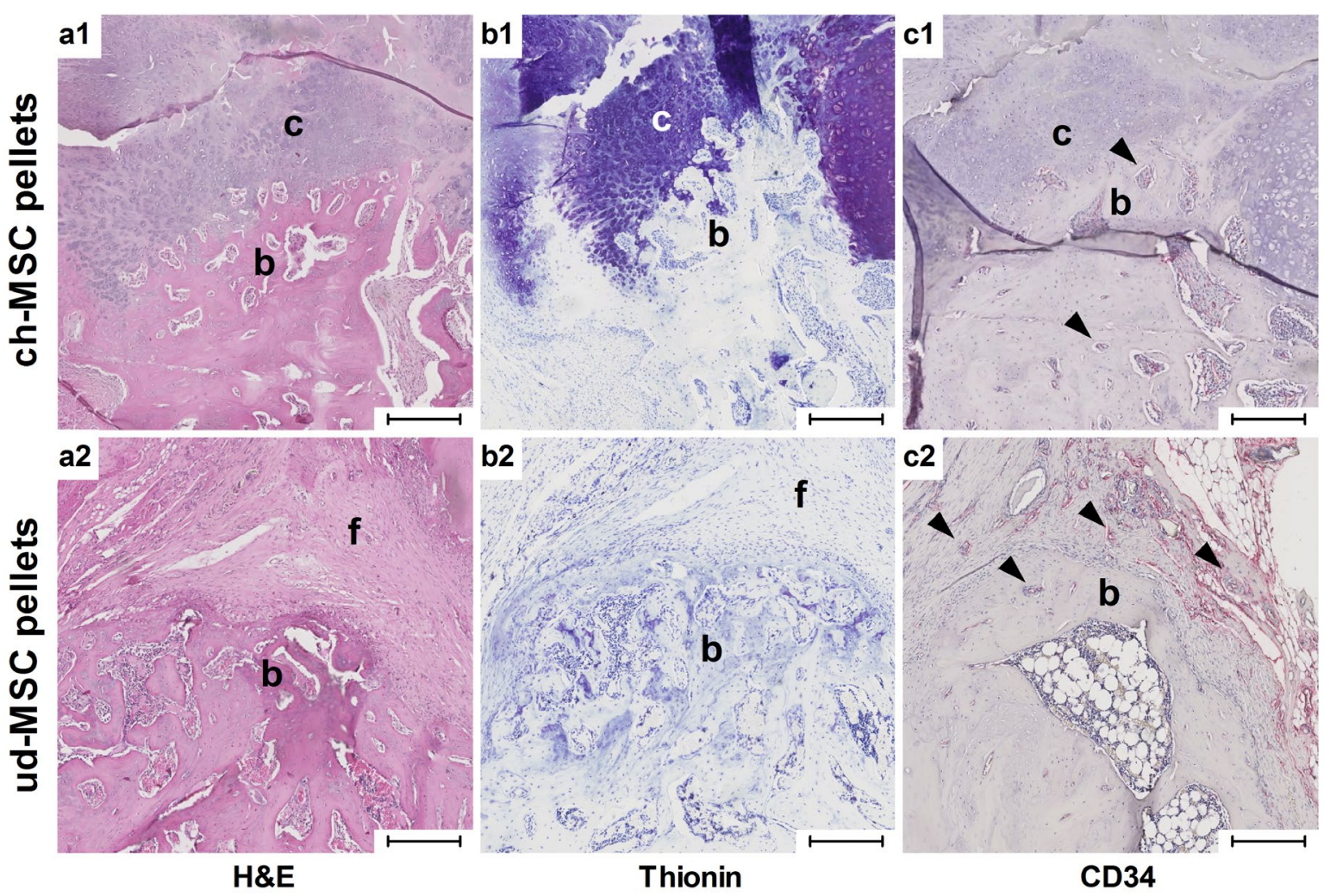

Fig. 9. Detailed views of the $6 \mathrm{~mm}$ bone defect grafted with ch-MSC pellets or ud-MSC pellets after 8 weeks, stained with respectively H\&E (a1-2), thionin (b1-2) and CD34 immunostaining (c1-2). Bone tissue is indicated with 'b', cartilage tissue is indicated with 'c' and fibrous tissue is indicated with ' $\mathrm{f}$ '. Arrows indicate vessel forming activity. Bar indicates $250 \mu \mathrm{m}$.
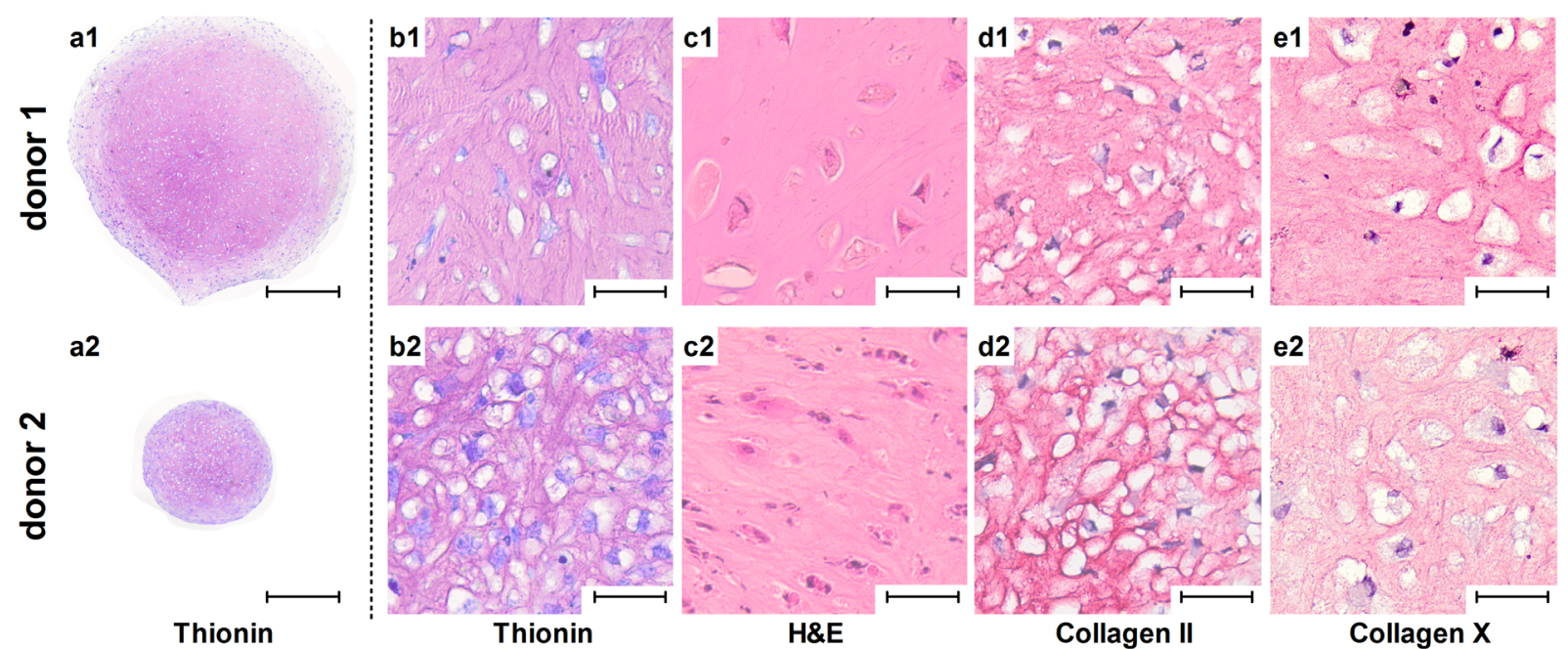

Fig. 10. Histology of ch-MSC pellets of donor 1 and 2 showing that ch-MSC pellets of donor 1 were bigger than ch-MSC pellets of donor 2 (a1-2). Bar indicates $500 \mu \mathrm{m}$. Detailed views showed that ch-MSC pellets of donor 1 formed a more structured extracellular matrix (b1-2 and c1-2), although the extracellular matrix of both donors contained collagen II (d1-2) and collagen X (e1-2), indicating that MSC of both donors had undergone hypertrophic chondrogenic differentiation. Bar indicates $50 \mu \mathrm{m}$.

fibrous tissue, vessel-forming activity was clearly observed (Fig. 9, c2: arrows). In both groups, bone observed on histology showed a good correlation with bone seen on corresponding micro-CT images (Fig. 8, c1-2).

Ch-MSC pellets of donor 2, which did not stimulate bone regeneration (Fig. 5), were smaller in size $(\sim 0.5 \mathrm{~mm})$ and contained more closely packed cells than ch-MSC pellets of donor 1 (Fig. 10, a1-2) or donor 3. Cell nuclei of ch-MSC pellets of donor 2 seemed viable (Fig. 10, c2), but the cell nuclei of ch-MSC pellets of donor 1 and donor 3 were smaller or had shrunk and more empty cell lacunae were observed throughout the extracellular matrix (Fig. 10, c1). Ch-MSC pellets of donor 1 and 2 had both undergone chondrogenic differentiation and consisted of hypertrophic chondrocytes (Fig. 10, d1-2 and e1-2), but the extracellular matrix of ch-MSC pellets from donor 2 

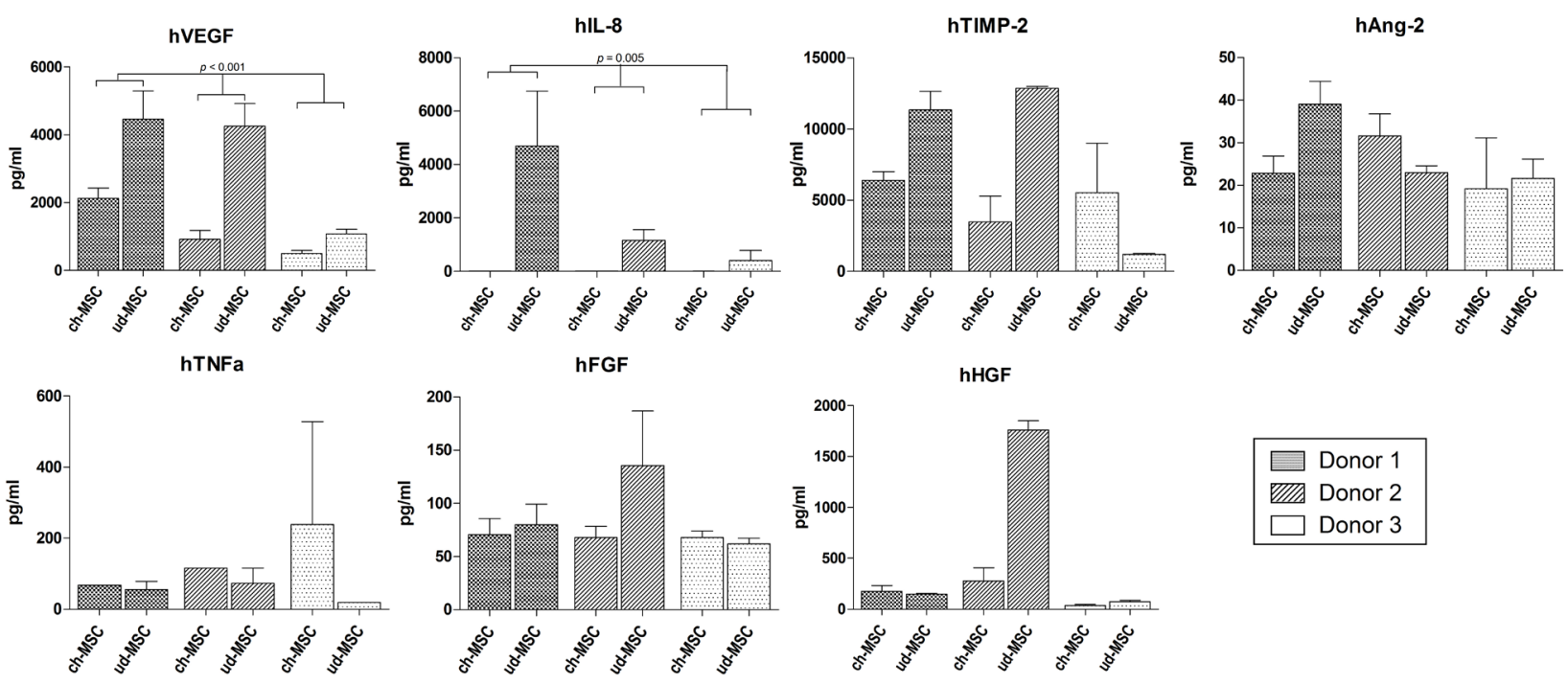

Fig. 11. Angiogenic cytokines released from ch-MSC and ud-HTC pellets in culture medium retrieved before grafting the bone defects.

appeared less structured than the extracellular matrix of ch-MSC pellets from donor 1 (Fig. 10, b1-2).

\section{Release of angiogenic cytokines}

Release of hTIMP-1 and PDGF-BB is not shown because release of hTIMP-1 exceeded the upper quantification limit $(3000 \mathrm{pg} / \mathrm{mL})$ and release of PDGF-BB did not exceed the lower quantification limit $(1000 \mathrm{pg} / \mathrm{mL})$ of the assay. ChMSC pellets of all three donors released hVEGF, hTIMP-2, hAng-2, hTNFa, hFGF, and hHGF (Fig. 11). Cytokine release of ch-MSC pellets of donor 2 was not different from the cytokine release of the ch-MSC pellets of donor 1 and 3. Compared to ud-MSC pellets, less VEGF was released by ch-MSC pellets and no hIL-8 release was observed.

\section{Discussion}

Grafting bone defects and atrophic non-unions with MSCbased grafts is believed to hold great potential (Huey et al., 2012), but so far clinical results are rather disappointing (Steinert et al., 2012). Therefore, instead of stimulating intramembranous ossification with undifferentiated or osteogenically differentiated MSCs, some papers advocate to stimulate endochondral ossification with chondrogenically differentiated MSCs (Farrell et al., 2009; Oliveira et al., 2009b; Scotti et al., 2010). In our study, grafting of critical femoral bone defects that were left untreated for 6 weeks with chondrogenically differentiated MSC pellets was found to result in significantly more bone than grafting with undifferentiated MSC pellets (Figs. 3 and 5) and we showed that this bone was formed through endochondral ossification (Fig. 6). Grafting with chondrogenically differentiated MSC pellets can result in bone regeneration capable of completely bridging the defect (Fig. 4). However, the effect of chondrogenically differentiated MSC pellets is donor-depend (Fig. 5).

The complex orthotopic environments that can be encountered during insufficient or impaired fracture repair (Garcia et al., 2013) were simulated using a critical femoral bone defect model that was grafted after 6 weeks. During the first 6 weeks, untreated defects formed an atrophic non-union and the remaining fracture gap was filled with fibrous tissue (Fig. 1c). Grafting these defects with chondrogenically differentiated MSC pellets resulted in large amounts of bone formation through endochondral ossification (Fig. 5b). Although extensive bone regeneration was observed in defects grafted with chondrogenically differentiated MSC pellets of donor 1 and 3 , only one defect was completely bridged after 8 weeks (Fig. 4). The inability to bridge the other defect is may be explained by a suboptimal method of fixation, because the micro-CT images of these defects show the formation of a hypertrophic non-union with a typical horse-shoe or elephant-foot configuration (Figs. 5 and 8) (Naimark et al., 1981), and the remaining fracture gap contained cartilage tissue (Fig. 8 and 9). Although a longer follow-up might have resulted in union, hypertrophic non-unions are usually well treated by providing increased stability over the nonunion (Megas, 2005).

Similar to previous ectopic implantations (Jukes et al., 2008; Oliveira et al., 2009b; Scotti et al., 2010; Farrell et al., 2011), we used immunocompromised animals to avoid host-versus-graft reactions against human MSCs. This raised the relevant question whether chondrogenically differentiated MSC pellets remain capable of initiating endochondral ossification in an immunocompetent host. Chondrogenically differentiated MSCs of rat origin have shown to remain capable of initiating endochondral ossification when implanted in immunocompetent rats (Farrell et al., 2006; Farrell et al., 2011). The use of rat MSCs makes it also possible to gain more insight in the influence of a specific immune response of the host to implanted chondrogenically differentiated MSC pellets. Specific immune responses may be the driving factor in the bone regeneration observed, because during endochondral ossification bone regeneration is preceded by osteoclast-mediated degradation of the cartilage template. Osteoclasts are monocyte-derived cells. Furthermore, chondrogenically differentiated MSC pellets that were 
exposed to IL-1, a pro-inflammatory cytokine, showed enhanced accumulation of MMP-13 and increase levels of released stromal cell-derived factor-1, which resulted in more osteoclast recruitment and faster bone regeneration (Scotti et al., 2013).

Obtaining high yields of MSCs is important for generating sufficient chondrogenically differentiated MSC pellets to be used in clinical applications. MSCs that are capable of undergoing chondrogenic differentiation can be obtained from various tissues including bone marrow, fat, muscle, synovium, periosteum (Sakaguchi et al., 2005). Bone marrow derived MSCs, used here, have more chondrogenic potential than fat derived MSCs (Winter et al., 2003) or muscle derived MSCs (Sakaguchi et al., 2005). Bone marrow derived MSC can be obtained by a simple percutaneous procedure, and the yield of MSCs obtained can be improved when a concentration device is used that centrifuges a larger volume of bone marrow (Hernigou et al., 2005). Furthermore, the proliferation of obtained MSC can be enhanced by supplementing in vitro culture media with FGF-2, or other factors such as platelet-derived growth factor, ascorbic acid and epidermal growth factor (Gharibi and Hughes, 2012). More MSCs result in a higher volume of chondrogenically differentiated MSC pellets that can be generated. But with the culturing protocol that we used here, we were able to generate around 200 chondrogenically differentiated MSC pellets per donor. Theoretically this can result in $2.7 \mathrm{~cm}^{3}$ pellet volume, and is similar to volumes in which commercially available bone substitutes are sold (Van der Stok et al., 2013c). Although this volume may not be sufficient or requires the use of additional bone substitutes to graft large bone defects in humans $(>2 \mathrm{~cm})$, it might already be enough to graft the much smaller fracture gap that is typically seen in an atrophic non-unions (<2 mm) (Naimark et al., 1981).

VEGF is known to play a key role in endochondral ossification (Patil et al., 2012) and the release of VEGF could also be to the driving factor through which chondrogenically differentiated MSC pellets can initiate endochondral ossification. VEGF was indeed produced and released by chondrogenically differentiated MSC pellets of all three donors (Fig. 11), but endochondral ossification did not occur after grafting with chondrogenically differentiated MCS pellets of donor 2 (Fig. 5). The release of VEGF from chondrogenically differentiated MSC pellets was also less than from undifferentiated MSC pellets at the moment of implantation (Fig. 11). The cumulative release of VEGF from the chondrogenically differentiated MSC pellets, however, might still be superior after in vivo implantation due to prolonged cell survival in low-oxygen environment of the hypertrophic chondrocytes. But the CD34 immunostaining performed on grafted defects after 7 and 14 (Figs. 6 and 7) and after 8 weeks (Fig. 9) did not suggest that a lot of vesselforming activity was taking place around chondrogenically differentiated MSC pellets. Vessel formation, however, may have been more controlled and synchronised with the bone formation compared to vessel formation upon grafting with undifferentiated pellets. This possible spatiotemporal role of VEGF in endochondral ossification upon grafting with chondrogenically differentiated MSC pellets warrants further investigation in longitudinal in vivo setting.

Bone regeneration was after grafting bone defects with chondrogenically differentiated MSC pellets was donordepend (Fig. 5), and this has also been observed after subcutaneous implantation (Farrell et al., 2009; Farrell et al., 2011). The non-potent chondrogenically differentiated MSC pellets of donor 2 did contain hypertrophic chondrocytes that produced VEGF, similar as the potent chondrogenically differentiated MSC pellets of donor 1 and 3 (Fig. 8) and there was no clear difference in the release of angiogenic cytokines between the donors (Fig. 11). The only difference between the non-potent and the potent chondrogenically differentiated MSC pellets that we could find was their size (Fig. 8), non-potent pellets were smaller and contained less extracellular matrix. This may indicate an important role for the extracellular matrix that is generated by chondrogenically differentiated MSC pellets, but on the other hand it has also been suggested that hypertrophic chondrocytes undergoes a transformation into bone (Scotti et al., 2010; Farrell et al., 2011; Bahney et al., 2013;). Determining the exact composition of the extracellular matrix generated by chondrogenically differentiated MSC pellets of a larger number of different donors followed by an in vivo implantation to assess their potency to form bone and to determine the survival of these hypertrophic chondrocytes in vivo may provide valuable new insights in the mechanism through with chondrogenically differentiated MSCs initiate endochondral ossification and may help to develop protocols to generate potent pellets of each donor.

\section{Conclusion}

In conclusion, grafting critical femoral bone defects that were left empty to establish an atrophic non-union with chondrogenically differentiated MSC pellets leads to significantly more bone regeneration than grafting with undifferentiated MSC pellets in athymic rats. With chondrogenically differentiated MSC pellets, bone regenerates rapidly through endochondral ossification, and through bone remodelling leading to restoration of the cortex and the intramedullary space. When this MSCbased approach can be optimised such that sufficient and potent chondrogenically differentiated MSC pellets can be generated for each single patient, grafting with chondrogenically differentiated MSC pellets may become a successful clinical treatment for bone repair in atrophic non-unions and large bone defects.

\section{Acknowledgements}

This research forms part of the Project P2.04 BONE-IP of the research program of the BioMedical Materials institute, co-funded by the Dutch Ministry of Economic Affairs and was supported by a grant from the Dutch government to the Netherlands Institute for Regenerative Medicine (NIRM, grant No. FES0908). We thank Dr. R.J. Rottier (Department of Pediatric Surgery, Erasmus MC-Sophia Children's Hospital, Rotterdam, The Netherlands) for the biotechnical support. 


\section{References}

Bahney CS, Hu DP, Taylor AJ, Ferro F, Britz HM, Hallgrimsson B, Johnstone B, Miclau T, Marcucio RS (2013) Stem cell derived endochondral cartilage stimulates bone healing by tissue transformation. J Bone Miner Res. doi: 10.1002/jbmr.2148.

Banwart JC, Asher MA, Hassanein RS (1995) Iliac crest bone graft harvest donor site morbidity. A statistical evaluation. Spine (Phila Pa 1976) 20: 1055-1060.

Brighton CT, Krebs AG (1972) Oxygen tension of healing fractures in the rabbit. J Bone Joint Surg Am 54: 323-332.

Carter DR, Beaupre GS, Giori NJ, Helms JA (1998) Mechanobiology of skeletal regeneration. Clin Orthop Relat Res 355 Suppl: S41-55.

Chatterjea A, van der Stok J, Danoux CB, Yuan H, Habibovic P, van Blitterswijk CA, Weinans H, de Boer J (2013) Inflammatory response and bone healing capacity of two porous calcium phosphate ceramics in critical size cortical bone defects. J Biomed Mater Res A. doi: 10.1002/ jbm.a.34815.

Claes L, Recknagel S, Ignatius A (2012) Fracture healing under healthy and inflammatory conditions. Nat Rev Rheumatol 8: 133-143.

Cypher TJ, Grossman JP (1996) Biological principles of bone graft healing. J Foot Ankle Surg 35: 413-417.

Dimitriou R, Mataliotakis GI, Angoules AG, Kanakaris NK, Giannoudis PV (2011) Complications following autologous bone graft harvesting from the iliac crest and using the RIA: a systematic review. Injury 42 Suppl 2: S3-15.

Einhorn TA (1995) Enhancement of fracture-healing. J Bone Joint Surg Am 77: 940-956.

Farrell E, O’Brien FJ, Doyle P, Fischer J, Yannas I, Harley BA, O’Connell B, Prendergast PJ, Campbell VA (2006) A collagen-glycosaminoglycan scaffold supports adult rat mesenchymal stem cell differentiation along osteogenic and chondrogenic routes. Tissue Eng 12: 459468.

Farrell E, van der Jagt OP, Koevoet W, Kops N, van Manen CJ, Hellingman CA, Jahr H, O'Brien FJ, Verhaar JA, Weinans H, van Osch GJ (2009) Chondrogenic priming of human bone marrow stromal cells: a better route to bone repair? Tissue Eng Part C Methods 15: 285-295.

Farrell E, Both SK, Odorfer KI, Koevoet W, Kops N, O'Brien FJ, Baatenburg de Jong RJ, Verhaar JA, Cuijpers V, Jansen J, Erben RG, van Osch GJ (2011) In-vivo generation of bone via endochondral ossification by in-vitro chondrogenic priming of adult human and rat mesenchymal stem cells. BMC Musculoskelet Disord 12: 31.

Garcia P, Histing T, Holstein JH, Klein M, Laschke MW, Matthys R, Ignatius A, Wildemann B, Lienau J, Peters A, Willie B, Duda G, Claes L, Pohlemann T, Menger MD (2013) Rodent animal models of delayed bone healing and non-union formation: a comprehensive review. Eur Cell Mater 26: 1-14.

Gawlitta D, Farrell E, Malda J, Creemers LB, Alblas J, Dhert WJ (2010) Modulating endochondral ossification of multipotent stromal cells for bone regeneration. Tissue Eng Part B Rev 16: 385-395.
Gharibi B, Hughes FJ (2012) Effects of medium supplements on proliferation, differentiation potential, and in vitro expansion of mesenchymal stem cells. Stem Cells Transl Med 1: 771-782.

Giannoudis PV, Einhorn TA, Marsh D (2007) Fracture healing: the diamond concept. Injury 38 Suppl 4: S3-6.

Hernigou P, Poignard A, Beaujean F, Rouard H (2005) Percutaneous autologous bone-marrow grafting for nonunions. Influence of the number and concentration of progenitor cells. J Bone Joint Surg Am 87: 1430-1437.

Hierck BP, Iperen LV, Gittenberger-De Groot AC, Poelmann RE (1994) Modified indirect immunodetection allows study of murine tissue with mouse monoclonal antibodies. J Histochem Cytochem 42: 1499-1502.

Huey DJ, Hu JC, Athanasiou KA (2012) Unlike bone, cartilage regeneration remains elusive. Science 338: $917-$ 921.

Jukes JM, Both SK, Leusink A, Sterk LM, van Blitterswijk CA, de Boer J (2008) Endochondral bone tissue engineering using embryonic stem cells. Proc Natl Acad Sci U S A 105: 6840-6845.

Kronenberg HM (2003) Developmental regulation of the growth plate. Nature 423: 332-336.

Lacroix D, Prendergast PJ (2002) A mechanoregulation model for tissue differentiation during fracture healing: analysis of gap size and loading. J Biomech 35: 1163-1171.

Megas P (2005) Classification of non-union. Injury 36 Suppl 4: S30-37.

Naimark A, Miller K, Segal D, Kossoff J (1981) Nonunion. Skeletal Radiol 6: 21-25.

Oliveira SM, Amaral IF, Barbosa MA, Teixeira CC (2009a) Engineering endochondral bone: in vitro studies. Tissue Eng Part A 15: 625-634.

Oliveira SM, Mijares DQ, Turner G, Amaral IF, Barbosa MA, Teixeira CC (2009b) Engineering endochondral bone: in vivo studies. Tissue Eng Part A 15: 635-643.

Patil AS, Sable RB, Kothari RM (2012) Occurrence, biochemical profile of vascular endothelial growth factor (VEGF) isoforms and their functions in endochondral ossification. J Cell Physiol 227: 1298-1308.

Quarto R, Mastrogiacomo M, Cancedda R, Kutepov SM, Mukhachev V, Lavroukov A, Kon E, Marcacci M (2001) Repair of large bone defects with the use of autologous bone marrow stromal cells. N Engl J Med 344: 385-386.

Sakaguchi Y, Sekiya I, Yagishita K, Muneta T (2005) Comparison of human stem cells derived from various mesenchymal tissues: superiority of synovium as a cell source. Arthritis Rheum 52: 2521-2529.

Scotti C, Tonnarelli B, Papadimitropoulos A, Scherberich A, Schaeren S, Schauerte A, Lopez-Rios J, Zeller R, Barbero A, Martin I (2010) Recapitulation of endochondral bone formation using human adult mesenchymal stem cells as a paradigm for developmental engineering. Proc Natl Acad Sci USA 107: 7251-7256.

Scotti C, Piccinini E, Takizawa H, Todorov A, Bourgine P, Papadimitropoulos A, Barbero A, Manz MG, Martin I (2013) Engineering of a functional bone organ through endochondral ossification. Proc Natl Acad Sci USA 110: 3997-4002. 
Shapiro F (2008) Bone development and its relation to fracture repair. The role of mesenchymal osteoblasts and surface osteoblasts. Eur Cell Mater 15: 53-76.

Steinert AF, Rackwitz L, Gilbert F, Noth U, Tuan RS (2012) Concise review: the clinical application of mesenchymal stem cells for musculoskeletal regeneration: current status and perspectives. Stem Cells Transl Med 1: 237-247.

Van der Stok J, Van Lieshout EM, El-Massoudi Y, Van Kralingen GH, Patka P (2011) Bone substitutes in the Netherlands - a systematic literature review. Acta Biomater 7: 739-750.

Van der Stok J, Van der Jagt OP, Amin Yavari S, De Haas MF, Waarsing JH, Jahr H, Van Lieshout EM, Patka P, Verhaar JA, Zadpoor AA, Weinans H (2013a) Selective laser melting-produced porous titanium scaffolds regenerate bone in critical size cortical bone defects. J Orthop Res 31: 792-799.

Van der Stok J, Wang H, Amin Yavari S, Siebelt M, Sandker M, Waarsing JH, Verhaar JA, Jahr H, Zadpoor AA, Leeuwenburgh SC, Weinans H (2013b) Enhanced bone regeneration of cortical segmental bone defects using porous titanium scaffolds incorporated with colloidal gelatin gels for time- and dose-controlled delivery of dual growth factors. Tissue Eng Part A 19: 2605-2614.

Van der Stok J, Weinans H, Kops N, Siebelt M, Patka P, Van Lieshout EM (2013c) Properties of commonly used calcium phosphate cements in trauma and orthopaedic surgery. Injury 44:1368-1374.

Winter A, Breit S, Parsch D, Benz K, Steck E, Hauner H, Weber RM, Ewerbeck V, Richter W (2003) Cartilagelike gene expression in differentiated human stem cell spheroids: a comparison of bone marrow-derived and adipose tissue-derived stromal cells. Arthritis Rheum 48: 418-429.

\section{Discussion with Reviewers}

I. Martin: No empty control was incorporated into the study. Why was this? Figure 1 indicates that a certain amount of spontaneous repair had occurred during the initial 6 weeks. Do the authors know if bridging would occur if these defects are left untreated for 14 weeks $(6+8$ weeks)? Is there data from this or earlier studies from your lab using this model to confirm this is a critical sized defect?

Authors: The critical size of this bone defect using this fixation method has been confirmed by our group in two published studies (Chatterjea et al., 2013; van der Stok et al., 2013a, text references). In these studies, untreated defects did not show spontaneous bridging up to 12 weeks. Furthermore, in an unpublished study (Koolen et al., in preparation) we have also included an empty control group that was followed for 16 weeks. No spontaneous bridging was observed.
An empty control group was not included since our primary aim was to determine the regenerative capacity of chondrogenic differentiated MSCs in an established defect. The $6 \mathrm{~mm}$ defect was left untreated for 6 weeks. There was only little bone formation and after this period the defect is filled with fibrous tissue. The best control to address our primary research question is putting undifferentiated MSCs in the defect. To make this feasible without using scaffolds a three-day incubation was needed so that transplantable pellets were formed. Having empty controls in this established non-union model, would not provide further data with regard to our research question. The little bone formation that is indeed notable, but in regard to the chMSC pellets it is very minor. Previous studies did examine the effects of undifferentiated and osteogenic differentiated MSCs. This strategy has little success, please see Steinert et al. (2012) (text reference). This is the primary reason to examine new strategies.

I. Martin: What was the logic for implanting 6 pellets per defect?

Authors: We were able to completely fill the defect using 6 pellets, this is shown in Fig. 1. Although you only can see 5 pellets, the defect was nicely filled.

I. Martin: Was the repair 6 weeks after implantation of ud-MSCs worse than that at the start of the study (i.e., 6 weeks after the defect was originally created)? Comparing Fig. 3 to Fig. 1 might suggest it is.

Authors: Indeed the reader might get this the impression when only looking at Figs. 1 and 3, but when you also take the micro-CT scans of Fig. 5a into account, one can also observe fairly good bone regeneration 8 weeks after implantation of ud-MSCs. So we think it is more or less equal.

I. Martin Other studies that incorporate undifferentiated MSCs into a ceramic scaffold or similar have demonstrated the utility of this approach. Could the absence of a supporting scaffold (and the cues it provides to MSCs) be the reason for the poor repair observed with ud-MSCs? This is an important question, as having to chondrogenically prime MSCs for 3 weeks prior to implantation is obviously an additional hurdle to be overcome for clinical translation. Authors: The absence of a supporting scaffold is likely to have a negative effect on ud-MSC pellets, but this is also true for the ch-MSC pellets. Using a suitable scaffold for additional mechanical support with ch-MSC pellets may help to avoid the formation of hypertrophic nonunion. To design scaffolds such that, next to providing mechanical support, they also provide clues to the MSCs is very interesting. Based on our results, we then suggest providing clues that stimulate chondrogenic differentiation of MSCs, because this can initiate bone regeneration through endochondral ossification and that appears to be very fast and efficient. 